\title{
About Twistor Spinors with Zero in Lorentzian Geometry ${ }^{\star}$
}

\author{
Felipe LEITNER
}

Universität Stuttgart, Institut für Geometrie und Topologie, Fachbereich Mathematik, Pfaffenwaldring 57, D-70550 Stuttgart, Germany

E-mail: leitner@mathematik.uni-stuttgart.de

URL: http://www.igt.uni-stuttgart.de/LstDiffgeo/Leitner/

Received April 06, 2009, in final form July 10, 2009; Published online July 28, 2009

doi:10.3842/SIGMA.2009.079

\begin{abstract}
We describe the local conformal geometry of a Lorentzian spin manifold $(M, g)$ admitting a twistor spinor $\varphi$ with zero. Moreover, we describe the shape of the zero set of $\varphi$. If $\varphi$ has isolated zeros then the metric $g$ is locally conformally equivalent to a static monopole. In the other case the zero set consists of null geodesic(s) and $g$ is locally conformally equivalent to a Brinkmann metric. Our arguments utilise tractor calculus in an essential way. The Dirac current of $\varphi$, which is a conformal Killing vector field, plays an important role for our discussion as well.
\end{abstract}

Key words: Lorentzian spin geometry; conformal Killing spinors; tractors and twistors

2000 Mathematics Subject Classification: 53C27; 53B30

\section{Introduction}

There are two conformally covariant first order partial differential equations for spinors, the Dirac equation and the twistor equation. The twistor equation is an overdetermined system of PDE's, which was first introduced by R. Penrose in the context of General Relativity (cf. [24]). We are interested in this article in solutions of the twistor equation with zeros on Lorentzian spin manifolds.

Let $\left(M^{n}, g\right)$ be a time-oriented Lorentzian manifold of dimension $n \geq 3$ with spin structure and spinor bundle $\mathcal{S}$. The twistor equation for spinors $\varphi \in \Gamma(\mathcal{S})$ is given by

$$
\nabla_{X}^{\mathcal{S}} \varphi+\frac{1}{n} X \cdot \mathcal{D} \varphi=0 \quad \text { for any } \quad X \in T M
$$

(cf. Section 2). Its solutions are called twistor spinors or conformal Killing spinors. We prefer the latter notion in this article (apart from the title and abstract).

There are many geometric structure results for conformal Killing spinors in the literature (cf. e.g. [22, 21, 5, 6] and the surveys [3, 4]). Especially, the case of Riemannian geometry is well studied. In particular, it is known that a Riemannian metric admitting a conformal Killing spinor with zero is conformally equivalent to an asymptotically locally Euclidean and Ricciflat metric. This result is achieved by conformally rescaling the metric with the norm of the conformal Killing spinor. The aim of this article is to derive an analogous result in Lorentzian geometry. In particular, we study in the following the local conformal geometry and the shape of the zero set of conformal Killing spinors on Lorentzian manifolds. However, an analogous treatment of the problem as in Riemannian geometry is not possible for various reasons. Instead

\footnotetext{
*This paper is a contribution to the Special Issue "Élie Cartan and Differential Geometry". The full collection is available at http://www.emis.de/journals/SIGMA/Cartan.html
} 
we employ in this article methods of tractor calculus and its spinorial counterpart - twistor calculus (cf. $[25,1,20]$ ). This approach and its results demonstrate the force of tractor (and twistor) calculus in a particular instance.

Solutions of the twistor equation with zeros on Lorentzian manifolds are interesting, in particular, for the following reason. A conformal Killing spinor $\varphi$ is always accompanied by a conformal Killing vector field $V_{\varphi}$, the so-called Dirac current. If the conformal Killing spinor $\varphi$ admits a zero, then the Dirac current $V_{\varphi}$ is an essential conformal Killing vector field. In particular, the local flow of $V_{\varphi}$ consists of conformal transformations, which do not preserve any metric in the underlying conformal class! A conjecture by A. Lichnerowicz states that any compact Lorentzian manifold with an essential conformal transformation group is conformally flat (cf. [10]). In the analytic category Ch. Frances proved in [11] that any Lorentzian manifold, admitting a causal conformal Killing vector with a zero, is conformally flat. On the other hand, we have constructed in [19] a Lorentzian spin manifold admitting a conformal Killing spinor with isolated zero, which is not conformally flat in the spacelike neighbourhood of the isolated zero. This is possible, since the construction in [19] is not analytic, but only $C^{1}$-differentiable.

In Section 2 we recall the twistor equation in the classical way as partial differential equation for spinor fields. In Section 3 we introduce conformal tractor and twistor calculus via Cartan geometry. This approach allows us to reformulate the Penrose twistor equation in terms of parallel twistor fields. Spinors and twistors are accompanied by so-called spinor and twistor squares, respectively. In Lorentzian geometry the spinorial square of first degree plays a particular role. This is the so-called Dirac current. For conformal Killing spinors $\varphi$ the Dirac current $V_{\varphi}$ is a conformal Killing vector field. On the other hand, to $\varphi$ and $V_{\varphi}$ there corresponds a parallel twistor with adjoint tractor. All this is explained in Section 4. The tractor/twistor picture is the essential tool for the geometric description of the underlying Lorentzian conformal structure. Brinkmann spaces and static monopole solutions are the possible underlying geometries. This is stated in Proposition 1 of Section 5. Finally, in Section 6 we discuss the shape of the zero set $\operatorname{zero}(\varphi)$ and the singular set $\operatorname{sing}(\varphi)$ of a conformal Killing spinor $\varphi$. Either isolated zeros or null geodesics are possible. These results about the shape are based on investigations of [15] (see also [16]).

\section{The twistor equation for spinors}

We briefly recall here the twistor equation for spinors (in Lorentzian geometry), which is most notably a conformally covariant, overdetermined partial differential equation of first order on manifolds (cf. [24, 5]).

Let $\mathrm{SO}(r, s)$ denote the special orthogonal group, which acts by the standard representation on the Euclidean space $\mathbb{R}^{r, s}=\left(\mathbb{R}^{n},(\cdot, \cdot)_{r, s}\right)$ of dimension $n=r+s$ with (indefinite) scalar product of signature $(r, s)$ (where $r$ denotes the number of timelike vectors in an orthonormal basis of $\left.\mathbb{R}^{r, s}\right)$. The spin group of signature $(r, s)$ is denoted by $\operatorname{Spin}(r, s)$ and covers $\operatorname{SO}(r, s)$ twice by a group homomorphism

$$
\lambda: \operatorname{Spin}(r, s) \rightarrow \mathrm{SO}(r, s)
$$

Then let $\Delta_{r, s}$ denote the standard complex spinor module with spin representation $\rho=\rho_{r, s}$ (cf. e.g. $[14,2,5])$. We denote the connected components of the identity elements in $\mathrm{SO}(r, s)$ and $\operatorname{Spin}(r, s)$ by $\mathrm{SO}_{o}(r, s)$ and $\operatorname{Spin}_{o}(r, s)$, respectively.

Now let $\left(M^{n}, g\right)$ be a connected and time-oriented Lorentzian spin manifold (i.e. $r=1$ ) of dimension $n \geq 3$. The principal $\mathrm{SO}_{o}(1, n-1)$-bundle of time- and space-oriented orthonormal frames on $M$ is denoted by $S O(M)$. We fix a spin structure $(\operatorname{Spin}(M), \pi)$ on $M$, that is a $\lambda$ reduction of $S O(M)$ to the spin group $\operatorname{Spin}_{o}(1, n-1)$. The (complex) spinor bundle is given 
by

$$
\mathcal{S}=\operatorname{Spin}(M) \times_{\rho} \triangle_{1, n-1} .
$$

We denote the set of smooth sections in $\mathcal{S}$ by $\Gamma(\mathcal{S})$ and refer to them as spinor fields or just spinors on $(M, g)$. The spinor bundle $\mathcal{S}$ is equipped with an $\left(\operatorname{Spin}_{o}(1, n-1)\right.$-invariant) indefinite Hermitian product $\langle\cdot, \cdot\rangle_{\mathcal{S}}$. Moreover, the Levi-Civita connection $\omega^{g}$ on $S O(M)$ lifts via $\pi$ to a connection on the spin frame bundle $\operatorname{Spin}(M)$, which in turn induces a covariant derivative on $\mathcal{S}$, the so-called spinor derivative

$$
\nabla^{\mathcal{S}}: \Gamma(\mathcal{S}) \rightarrow \Omega^{1}(M) \otimes \Gamma(\mathcal{S}) .
$$

The spinor derivative $\nabla^{\mathcal{S}}$ preserves the Hermitian product $\langle\cdot, \cdot\rangle_{\mathcal{S}}$.

The bundle $T^{*} M \otimes \mathcal{S}$ of spinor-valued 1-forms decomposes into the direct sum $\mathcal{S} \oplus \mathcal{K}$, where $\mathcal{K}$ is the kernel of the Clifford multiplication of $T^{*} M$ on $\mathcal{S}$. The superposition of $\nabla^{\mathcal{S}}$ with the projection $\pi_{\mathcal{S}}$ onto $\mathcal{S}$ yields the Dirac operator $\mathcal{D}^{g}: \Gamma(\mathcal{S}) \rightarrow \Gamma(\mathcal{S})$ on $(M, g)$. The projection $\mathcal{P}^{g}:=\pi_{\mathcal{K}} \circ \nabla^{\mathcal{S}}: \Gamma(\mathcal{S}) \rightarrow \Gamma(\mathcal{K})$ is the so-called Penrose operator. The (non-trivial) elements of the kernel of the Penrose operator $\mathcal{P}$ are called twistor spinors, or alternatively, conformal Killing spinors. (Apart from the title we use in this article the latter notation.) It follows easily from the definition that a spinor field $\varphi \in \Gamma(\mathcal{S})$ is a conformal Killing spinor if and only if $\varphi$ satisfies the Penrose twistor equation

$$
\nabla_{X}^{S} \varphi+\frac{1}{n} X \cdot \mathcal{D} \varphi=0 \quad \text { for any } \quad X \in T M,
$$

where $X \cdot \mathcal{D} \varphi$ denotes the Clifford product of $X$ with the spinor $\mathcal{D} \varphi$.

In this article, we are interested in Lorentzian spin manifolds $\left(M^{n}, g\right), n \geq 3$, which admit a non-trivial solution $\varphi$ of (1) such that the zero set $\operatorname{zero}(\varphi)=\{p \in M \mid \varphi(p)=0\}$ is non-empty in $M$. In particular, we are interested in the shape of $\operatorname{zero}(\varphi)$ and the geometric properties of $\left(M^{n}, g\right)$ (locally outside of the zero set $\operatorname{zero}(\varphi)$ ).

The conformal class of a metric $g$ on $M$ consists of all those metrics $\tilde{g}$, which differ from $g$ only by multiplication with a positive function on $M$, i.e., $\tilde{g}=e^{2 f} g$ for some $f \in C^{\infty}(M)$. It is a well known fact that $\mathcal{D}$ and $\mathcal{P}$ are conformally covariant differential operators. In case of the Penrose operator $\mathcal{P}$ this means

$$
\mathcal{P}^{\tilde{g}}=e^{-f / 2} \circ \mathcal{P}^{g} \circ e^{-f / 2}
$$

for $\tilde{g}=e^{2 f} g$, i.e., the bidegree of $\mathcal{P}$ is $(-1 / 2,1 / 2)$. In particular, the kernel of $\mathcal{P}$ is a conformal invariant of $(M, c)$. The framework of conformal Cartan geometry provides a tool, in particular, for the invariant construction of the Penrose twistor equation. We aim to explain this next. This approach will be the key to our discussion of conformal Killing spinors with zeros on Lorentzian manifolds.

\section{Tractors and twistors}

We introduce here the standard tractor bundle $\mathcal{T}$ with tractor connection $\nabla^{\mathcal{T}}$ of conformal (Lorentzian) geometry via Cartan geometry (cf. [25, 1]). The spinorial counterpart is the socalled twistor bundle $\mathcal{W}$ with connection (cf. e.g. [20]). We then argue that conformal Killing spinors correspond uniquely to parallel sections of $\mathcal{W}$ (cf. $[23,12,5]$ ).

The projective null cone $\mathbb{P} L$ of the Euclidean space $\mathbb{R}^{2, n}$ of signature $(2, n)$ serves as the standard model of conformal Lorentzian geometry. As a homogeneous space, $\mathbb{P} L$ is given by $\mathrm{SO}_{o}(2, n) / P$, where $P \subset \mathrm{SO}_{o}(2, n)$ is the parabolic subgroup, which stabilises a null line in $\mathbb{R}^{2, n}$. The group $\mathrm{SO}_{o}(2, n)$ acts naturally by conformal transformations on $\mathbb{P} L$. 
Now let $\left(M^{n}, g\right)$ be a time- and space-oriented Lorentzian manifold of dimension $n \geq 3$. We denote the conformal class of $g$ on $M$ by $c=[g]$. Thus we obtain a conformal Lorentzian manifold $\left(M^{n}, c\right)$. The conformal structure $c=[g]$ on $M$ is equivalently described by a principal $P$-bundle $P(M)$ over $M$ with canonical Cartan connection

$$
\omega^{c}: P(M) \rightarrow \mathfrak{s o}(2, n)
$$

where $\mathfrak{s o}(2, n)$ denotes the orthogonal Lie algebra of signature $(2, n)$. The canonical Car$\tan$ connection $\omega^{c}$ is uniquely determined by the condition $\partial^{*} \kappa=0$, where $\kappa: P(M) \rightarrow$ $\operatorname{Hom}\left(\Lambda^{2} \mathbb{R}^{n}, \mathfrak{s o}(2, n)\right)$ denotes the curvature function of $\omega^{c}$ and $\partial^{*}$ is the Kostant-codifferential (cf. $[13,8])$.

The associated vector bundle

$$
\mathcal{T}:=P(M) \times_{\iota} \mathbb{R}^{2, n},
$$

where $\iota$ denotes the standard representation of $P \subset \mathrm{SO}_{o}(2, n)$ on $\mathbb{R}^{2, n}$, is called the standard tractor bundle of $(M, c)$ (cf. $[25,1])$. The tractor bundle $\mathcal{T}$ is equipped with an invariant metric $\langle\cdot, \cdot\rangle_{\mathcal{T}}$, and the Cartan connection $\omega^{c}$ induces a $\langle\cdot, \cdot\rangle_{\mathcal{T}}$-compatible covariant derivative $\nabla^{\mathcal{T}}$ on $\mathcal{T}$. Moreover, $\mathcal{T}$ admits a $P$-invariant filtration

$$
\mathcal{T} \supset \mathcal{T}^{0} \supset \mathcal{T}^{1}
$$

where $\mathcal{T}^{1}$ is the subbundle of $\mathcal{T}$, which corresponds to the $P$-stable null line of the standard representation on $\mathbb{R}^{2, n}$, and $\mathcal{T}^{0}$ is the $\langle\cdot, \cdot\rangle_{\mathcal{T}}$-orthogonal bundle to $\mathcal{T}^{1}$ in $\mathcal{T}$.

Note that any metric $g \in c$ in the conformal class on $M$ serves as a so-called Weyl structure in the sense of [7] and reduces the structure group of $P(M)$ to $\mathrm{SO}_{o}(1, n-1)$. This reduction of the structure group with respect to $g \in c$ causes a splitting of the filtration of $\mathcal{T}$ into a direct sum of the form $\mathbb{R} \oplus T M \oplus \mathbb{R}$, where the first summand corresponds to $\mathcal{T} / \mathcal{T}^{0}$ and the last summand to $\mathcal{T}^{1}$. We set $s_{-}:=(1,0,0)$ and $s_{+}:=(0,0,1)$. Any section in $\mathcal{T} \rightarrow M$ is given with respect to $g \in c$ by a triple $(a, V, b)=a s_{-}+V+b s_{+}$with $a, b \in C^{\infty}(M)$ and $V \in \mathfrak{X}(M)$. The tractor metric satisfies $\langle(a, V, b),(\tilde{a}, \tilde{V}, \tilde{b})\rangle_{\mathcal{T}}=a \tilde{b}+b \tilde{a}+g(V, \tilde{V})$.

In case $M$ is a spin manifold for some (hence all) $g \in c$, the conformal Cartan geometry $\left(P(M), \omega^{c}\right)$ over $M$ lifts to a conformal spin Cartan geometry $\left(\tilde{P}(M), \omega^{c}\right)$, where $\tilde{P}$ in $\operatorname{Spin}_{o}(2, n)$ is the preimage of $P$ under $\lambda: \operatorname{Spin}_{o}(2, n) \rightarrow \operatorname{SO}_{o}(2, n)$, and $\tilde{P}(M)$ is a principal $\tilde{P}$-bundle. We call the $\rho_{2, n}$-associated vector bundle

$$
\mathcal{W}=\tilde{P}(M) \times{ }_{\rho} \triangle_{2, n}
$$

the twistor bundle of the conformal Lorentzian spin manifold $(M,[g])$ (with fixed spin structure). The twistor bundle $\mathcal{W}$ is equipped with an indefinite Hermitian inner product $\langle\cdot, \cdot\rangle_{\mathcal{W}}$ and an $\omega^{c}$-induced covariant derivative $\nabla^{\mathcal{W}}$ preserving $\langle\cdot, \cdot\rangle_{\mathcal{W}}$. Moreover, we have a fiberwise Clifford multiplication $:: \mathcal{T} \otimes \mathcal{W} \rightarrow \mathcal{W}$ of tractors with twistors. There is also a natural subbundle $\mathcal{W}^{1 / 2}$ in $\mathcal{W}$, that is the bundle of twistors, which are annihilated by Clifford multiplication with the elements of the real line bundle $\mathcal{T}^{1}$. This defines a natural filtration $\mathcal{W} \supset \mathcal{W}^{1 / 2}$ on the twistor bundle, and the quotient bundle $\mathcal{W} / \mathcal{W}^{1 / 2}$ is naturally isomorphic to the spinor bundle $\mathcal{S}[-1 / 2]:=\mathcal{S} \otimes \mathcal{E}[-1 / 2]$ of conformal weight $-1 / 2$. Here $\mathcal{E}[w], w \in \mathbb{R}$, denotes the density bundle, which is the trivial real line bundle over $M$ associated to the representation $|\operatorname{det}|^{w / n}$ of the general linear group GL( $n)$.

Any metric $g \in c$ in the conformal class on $M$ reduces the structure group of $\tilde{P}(M)$ to $\operatorname{Spin}_{o}(1, n-1)$. Accordingly, the filtration $\mathcal{W} \supset \mathcal{W}^{1 / 2}$ splits into the direct sum

$$
\mathcal{W} \cong g \mathcal{S} \oplus \mathcal{S}
$$


where both, the quotient $\mathcal{W} / \mathcal{W}^{1 / 2}$ and the subbundle $\mathcal{W}^{1 / 2}$, become isomorphic to the spinor bundle $\mathcal{S}$ with respect to $g$ on $M$. Note that the invariant Hermitian product on $\mathcal{W}$ is given with respect to the $g$-corresponding splitting (3) by

$$
\langle(\varphi, \psi),(\tilde{\varphi}, \tilde{\psi})\rangle_{\mathcal{W}}=\frac{i}{\sqrt{2}}\left(\langle\varphi, \tilde{\psi}\rangle_{\mathcal{S}}-\langle\psi, \tilde{\varphi}\rangle_{\mathcal{S}}\right)
$$

Now let us consider the most obvious condition $\nabla^{\mathcal{W}} \Phi=0$ for a twistor $\Phi \in \Gamma(\mathcal{W})$. This equation means that $\Phi$ is a parallel twistor on $(M, c)$ with respect to the canonical connection. It is clear by construction that this is a conformally invariant condition for a twistor $\Phi$. With respect to any $g \in c$ and the induced splitting $\Phi=(\varphi, \psi)$ of $(3)$ the equation $\nabla{ }^{\mathcal{W}} \Phi=0$ is equivalent to the Penrose equation (1) for $\varphi$ and the equation $\nabla_{X}^{\mathcal{S}} \psi=\frac{1}{\sqrt{2}} \mathrm{P}^{g}(X) \cdot \varphi$ for any $X \in T M$, where $\mathrm{P}^{g}(X) \cdot \varphi$ is the Clifford product with the image of the Schouten operator $\mathrm{P}^{g}$ on $(M, g)$. The Schouten operator $\mathrm{P}^{g}: T M \rightarrow T M$ is defined by

$$
\mathrm{P}^{g}=\frac{1}{n-2}\left(\left.\frac{\mathrm{scal}^{g}}{2(n-1)} \mathrm{id}\right|_{T M}-\operatorname{Ric}^{g}\right)
$$

where $\operatorname{Ric}^{g}$ is the Ricci curvature operator and scal ${ }^{g}$ is the scalar curvature of $(M, g)$. On the other hand, any conformal Killing spinor $\varphi \in \Gamma(\mathcal{S})$ on $(M, g)$ satisfies the equation $\nabla_{X}^{\mathcal{S}} \mathcal{D} \varphi=$ $\frac{n}{2} \mathrm{P}^{g}(X) \cdot \varphi$. Thus a parallel twistor $\Phi$ on $(M, c)$ corresponds via the splitting (3) with respect to some $g \in c$ uniquely to a conformal Killing spinor $\varphi \in \Gamma(\mathcal{S})$ on $(M, g)$. The correspondence is explicitly given by

$$
\varphi \stackrel{g}{\longleftrightarrow} \Phi=\left(\varphi, \frac{\sqrt{2}}{n} \mathcal{D}^{g} \varphi\right)
$$

(cf. $[5,16]$ ). Also note that, with respect to a metric $g \in c$, the Penrose operator $\mathcal{P}^{g}$ is the first differential operator in the spinorial BGG sequence, which maps sections of $\mathcal{S}[-1 / 2]=\mathcal{W} / \mathcal{W}^{1 / 2}$ to sections of $\mathcal{K}[1 / 2]=\mathcal{K} \otimes \mathcal{E}[1 / 2]$ on $(M, c)$ (cf. [9]). This explains the bidegree in (2) and, in particular, conformal Killing spinors naturally have conformal weight $-1 / 2$.

\section{The Dirac current and its adjoint tractor}

We recall here the notion of the Dirac current of a spinor $\varphi$ on a Lorentzian spin manifold. On the other hand, any twistor $\Phi$ on a conformal Lorentzian spin manifold gives rise in a natural way to an adjoint (resp. 2-form) tractor.

In general, the spinor module $\Delta_{r, s}$ can be understood as a square root of the complexified exterior algebra $\Lambda_{\mathbb{C}}:=\Lambda\left(\mathbb{R}^{r, s}\right) \otimes \mathbb{C}$ of the Euclidean space $\mathbb{R}^{n}$ with scalar product $(\cdot, \cdot)_{r, s}$. To be concrete, as representation spaces of $\operatorname{Spin}(r, s)$ we have

$$
\begin{array}{ll}
\zeta: \triangle_{r, s} \otimes \Delta_{r, s} \cong \Lambda_{\mathbb{C}} & \text { for } n \text { even } \text { and } \\
\zeta: \triangle_{r, s} \otimes \Delta_{r, s} \cong \Lambda_{\mathbb{C}}^{\text {ev }} \cong \Lambda_{\mathbb{C}}^{\text {odd }} & \text { for } n \text { odd },
\end{array}
$$

where $\Lambda_{\mathbb{C}}^{\text {ev }}$ and $\Lambda_{\mathbb{C}}^{\text {odd }}$ denote the exterior products of even and odd degree, respectively. (The isomorphism $\Lambda_{\mathbb{C}}^{\text {ev }} \cong \Lambda_{\mathbb{C}}^{\text {odd }}$ is realised by the Hodge- $\star$ operator.) In particular, for any $\varphi \in \Delta_{r, s}$ and $0 \leq i \leq n$ we have the notion of a spinor square $\zeta^{i}(\varphi) \in \Lambda_{\mathbb{C}}^{i}$ of degree $i$, which is by definition the homogeneous component of degree $i$ of $\zeta(\varphi \otimes \varphi)$. Recall that the exterior algebras of $\mathbb{R}^{r, s}$ and its dual space $\mathbb{R}^{r, s *}$ are canonically identified via the metric $(\cdot, \cdot)_{r, s}$. Accordingly, we have the dual (complex) $i$-form to $\zeta^{i}(\varphi)$ on $\mathbb{R}^{r, s}$, which we denote by $\zeta_{i}(\varphi)$. 
Especially, in the Lorentzian case $r=1$, the (real) vector $\zeta^{1}(\varphi) \in \mathbb{R}^{1, n-1}$ is explicitly given via the relation

$$
\left(\zeta^{1}(\varphi), x\right)_{1, n-1}=-\langle x \cdot \varphi, \varphi\rangle_{1, n-1} \quad \text { for all } \quad x \in \mathbb{R}^{1, n-1},
$$

where $\langle\cdot, \cdot\rangle_{1, n-1}$ is the Hermitian product on the spinor module $\Delta_{1, n-1}$. The vector $\zeta^{1}(\varphi)$ is causal (i.e. null or timelike) and future-directed for any $\varphi \neq 0$ (cf. e.g. [2, 16]). Accordingly, we obtain for any spinor field $\varphi \in \Gamma(\mathcal{S})$ on some time-oriented Lorentzian spin manifold $\left(M^{n}, g\right)$ a vector field $V_{\varphi}$, which is uniquely determined by the relation $g\left(V_{\varphi}, X\right)=-\langle X \cdot \varphi, \varphi\rangle_{\mathcal{S}}$ for all $X \in T M$. We call $V_{\varphi} \in \mathfrak{X}(M)$ the Dirac current of the spinor $\varphi \in \Gamma(\mathcal{S})$ on the Lorentzian manifold $(M, g)$. The Dirac current $V_{\varphi}$ is a causal and future-directed vector field, whose zero set coincides with the zero set of $\varphi \in \Gamma(\mathcal{S})$ :

$$
\operatorname{zero}\left(V_{\varphi}\right)=\operatorname{zero}(\varphi) \quad \text { on } M \text {. }
$$

We denote the corresponding dual 1-form to $V_{\varphi}$ via $g$ by $\alpha_{\varphi} \in \Omega^{1}(M)$.

Now let $\left(M^{n}, c\right)$ be a time-oriented conformal Lorentzian spin manifold of dimension $n \geq 3$. Recall that we have the tractor bundle $\mathcal{T}$ with dual $\mathcal{T}^{*}$ and the twistor bundle $\mathcal{W}$ over $(M, c)$. Moreover, we have the adjoint tractor bundle $\mathcal{A}$ on $(M, c)$, which is the associated vector bundle to the adjoint representation of the parabolic subgroup $P$ in $\mathrm{SO}_{o}(2, n)$ :

$$
\mathcal{A}:=P(M) \times \operatorname{Ad} \mathfrak{s o}(2, n) .
$$

The adjoint tractor bundle $\mathcal{A}$ is canonically isomorphic to the bundle $\Lambda^{2} \mathcal{T}^{*}$ of 2 -form tractors on $(M, c)$. (In the following, we will not distinguish between adjoint and 2-form tractors.) Note that the squaring $\zeta$ induces for any twistor $\Phi \in \Gamma(\mathcal{W})$ a 2-form tractor $\alpha_{\Phi} \in \Gamma(\mathcal{A})$ on $(M, c)$. This 2 -form tractor is explicitly given by the relation

$$
\left\langle\alpha_{\Phi}, A\right\rangle_{\mathcal{T}}=-i\langle A \cdot \Phi, \Phi\rangle_{\mathcal{W}} \quad \text { for any } \quad A \in \mathcal{A}
$$

where $A \cdot \Phi$ denotes the Clifford product of a 2 -form tractor with a twistor and $\langle\cdot, \cdot\rangle_{\mathcal{T}}$ is the tractor metric extended to $\mathcal{A}$.

We have seen in the previous section that with respect to a Lorentzian metric $g \in c$ the tractor bundle $\mathcal{T}$ and the twistor bundle $\mathcal{W}$ split into direct sums over $M$. Also the adjoint tractor bundle $\mathcal{A}$ splits with respect to $g$ into a direct sum, which is

$$
T^{*} M \oplus \mathfrak{c o}(T M) \oplus T^{*} M,
$$

where $\mathfrak{c o}(T M)$ denotes the bundle of exterior 2-forms on $M$ plus multiples of the symmetric $(2,0)$-tensor $g$. Equivalently, using the dual tractors $s_{-}^{b}:=\left\langle s_{-}, \cdot\right\rangle_{\mathcal{T}}$ and $s_{+}^{b}:=\left\langle s_{+}, \cdot\right\rangle_{\mathcal{T}}$, any 2 -form tractor $A \in \Gamma(\mathcal{A})$ is given in the form

$$
s_{-}^{b} \wedge \alpha_{-}+\alpha_{0}+s_{-}^{b} \wedge s_{+}^{b} \wedge \alpha_{\mp}+s_{+}^{b} \wedge \alpha_{+}
$$

with uniquely determined differential forms $\alpha_{-}, \alpha_{+} \in \Omega^{1}(M), \alpha_{0} \in \Omega^{2}(M)$ and $\alpha_{\mp} \in C^{\infty}(M)$. We call $\alpha_{-}$the projecting component of $A$ (cf. [18]).

Now let $\Phi=(\varphi, \psi)$ be some twistor given as a pair of spinors with respect to the splitting (3) induced by $g \in c$. It is natural to ask how the twistor square $\alpha_{\Phi}$ and the spinor square $\alpha_{\varphi}$ (resp. the Dirac current $V_{\varphi}$ ) of the first component of $\Phi$ are related (with respect to $g \in c$ ). A straightforward calculation shows $\alpha_{\Phi}=\left(\alpha_{-}, \alpha_{0}, \alpha_{\mp}, \alpha_{+}\right)$with

$$
\alpha_{-}=\alpha_{\varphi}, \quad \alpha_{\mp}=\sqrt{2} \cdot \operatorname{Re}\langle\varphi, \psi\rangle_{s}, \quad \alpha_{+}=-\alpha_{\psi},
$$

and the 2 -form $\alpha_{0}$ on $M$ is determined by $\alpha_{0}(X, Y)=\frac{1}{\sqrt{2}} \operatorname{Re}\langle X \wedge Y \cdot \varphi, \psi\rangle_{\mathcal{S}}$ for $X, Y \in T M$. 
Now let $\varphi \in \Gamma(\mathcal{S})$ be a conformal Killing spinor on $\left(M^{n}, g\right)$. We have seen in the previous section that $\varphi$ corresponds in a unique way to a $\nabla^{\mathcal{W}}$-parallel twistor $\Phi \in \Gamma(\mathcal{W})$ on the conformal Lorentzian spin manifold $(M,[g])$, which is given with respect to $g$ by the pair $\Phi=\left(\varphi, \frac{\sqrt{2}}{n} \mathcal{D}^{g} \varphi\right)$. Note that, by construction, the induced 2 -form tractor $\alpha_{\Phi}$ of $\Phi$ on $(M,[g])$ is parallel with respect to the extended tractor connection $\nabla^{\mathcal{T}}$ on $\mathcal{A}$. In $[18,17]$ we have studied the projecting component of $\nabla^{\mathcal{T}}$-parallel $q$-form tractors, in general. The discussion shows that the projecting component of any $\nabla^{\mathcal{T}}$-parallel 2 -form tractor is a conformal Killing 1 -form $\alpha_{-}$, i.e., the symmetric trace-free part of the $(2,0)$-tensor $\nabla^{g} \cdot \alpha_{-}$is zero. Here $\nabla^{g}$ denotes the Levi-Civita connection of $(M, g)$. Equivalently, the Dirac current $V_{\varphi}$ of $\varphi$ is a conformal Killing vector, i.e., we have $\mathcal{L}_{V_{\varphi}} g=\lambda \cdot g$ for the Lie derivative $\mathcal{L}$ and some function $\lambda \in C^{\infty}(M)$. In fact, this property of the Dirac current of a conformal Killing spinor is well known (cf. e.g. [3])

On the other hand, we have also shown in [18] how to recover a $\nabla^{\mathcal{T}}$-parallel $q$-form tractor from its projecting component with respect to a choice of a metric $g$ in the conformal class. Here, in our case for the induced 2-form tractor $\alpha_{\Phi}$ of a $\nabla^{\mathcal{W}}$-parallel twistor $\Phi=\left(\varphi, \frac{\sqrt{2}}{n} \mathcal{D}^{g} \varphi\right)$, we have

$$
\begin{aligned}
& \alpha_{-}=\alpha_{\varphi}, \\
& \alpha_{\mp}=\frac{1}{n} d^{*} \alpha_{\varphi}=\frac{2}{n} \operatorname{Re}\langle\varphi, \mathcal{D} \varphi\rangle_{\mathcal{S}}, \\
& \alpha_{+}=\square \alpha_{\varphi}=\frac{-2}{n^{2}} \cdot \alpha_{\mathcal{D} \varphi},
\end{aligned}
$$

where $d^{*}$ denotes the codifferential and $\square$ is defined as $\frac{-1}{n-2}\left(\Delta^{g}-\operatorname{tr}_{g} \mathrm{P}^{g}\right)$ with Bochner-Laplacian $\Delta^{g}=\operatorname{tr}_{g}\left(\nabla^{g}\right)^{2}$. The 2 -form component of $\alpha_{\Phi}$ is given with respect to $X, Y \in T M$ by

$$
\alpha_{0}(X, Y)=\frac{1}{2} d \alpha_{\varphi}(X, Y)=\frac{1}{n} \operatorname{Re}\langle X \wedge Y \cdot \varphi, \mathcal{D} \varphi\rangle_{\mathcal{S}}
$$

\section{$5 \quad$ A local geometric description}

In this section we derive a local geometric description of conformal Lorentzian manifolds admitting a conformal Killing spinor (outside of its singular set). There occur two possible geometries, the so-called Brinkmann spaces and the static monopoles with parallel spinors. Our argument utilises tractor calculus in an essential way.

Let $\left(M^{n}, g\right), n \geq 3$, be a connected, time-oriented Lorentzian spin manifold with non-trivial conformal Killing spinor $\varphi \in \Gamma(\mathcal{S})$ and Dirac current $V_{\varphi}$. We have $\operatorname{zero}(\varphi)=\operatorname{zero}\left(V_{\varphi}\right)$, and $V_{\varphi}$ is outside of its zero set either null (i.e. $g\left(V_{\varphi}, V_{\varphi}\right)=0$ ) or timelike (i.e. $g\left(V_{\varphi}, V_{\varphi}\right)<0$ ) (cf. Lemma 2). If $V_{\varphi}$ is nowhere timelike on $M$, then we define the singular set of $\varphi$ by

$$
\operatorname{sing}(\varphi):=\operatorname{zero}(\varphi)
$$

If $V_{\varphi}$ is timelike in at least one point of $M$, then we set

$$
\operatorname{sing}(\varphi):=\operatorname{zero}\left(g\left(V_{\varphi}, V_{\varphi}\right)\right)
$$

Recall that the corresponding $\nabla^{\mathcal{W}}$-parallel twistor to $\varphi$ is given with respect to $g$ by $\Phi=$ $\left(\varphi, \frac{\sqrt{2}}{n} \mathcal{D} \varphi\right) \in \Gamma(\mathcal{W})$ and the induced 2-form tractor is $\alpha_{\Phi}=\left(\alpha_{-}, \alpha_{0}, \alpha_{\mp}, \alpha_{+}\right)$.

It is a matter of fact that the $\mathrm{SO}(2, n)$-module $\mathfrak{s o}(2, n) \cong \Lambda^{2}\left(\mathbb{R}^{2, n^{*}}\right)$ decomposes under the adjoint action into the disjoint union of $\mathrm{SO}(2, n)$-orbits. A distinguished element of such an $\mathrm{SO}(2, n)$-orbit can then be seen as a normal form for all the members of that orbit. Note that any fiber $\mathcal{A}_{p}, p \in M$, of the 2 -form tractor bundle $\mathcal{A}$ on $(M,[g])$ is uniquely identified with $\Lambda^{2}\left(\mathbb{R}^{2, n^{*}}\right)$ via the choice of a tractor frame at $p \in M$ (i.e. an orthonormal basis of $\mathcal{T}_{p}$ ). Via 
such an identification any 2 -form tractor in $\mathcal{A}_{p}$ belongs to a uniquely determined $\mathrm{SO}(2, n)$-orbit of $\Lambda^{2}\left(\mathbb{R}^{2, n^{*}}\right)$. If two 2-form tractors $A_{p} \in \mathcal{A}_{p}$ and $A_{q} \in \mathcal{A}_{q}, p, q \in M$, belong to the same orbit via an identification with $\Lambda^{2}\left(\mathbb{R}^{2, n^{*}}\right)$, we say $A_{p}$ and $A_{q}$ have the same orbit type. In our situation, the 2 -form tractor $\alpha_{\Phi}$, which belongs to the conformal Killing spinor $\varphi$ on $(M,[g])$, is $\nabla^{\mathcal{T}}$-parallel. Hence, since the tractor connection $\nabla^{\mathcal{T}}$ is induced by a Cartan connection with values in $\mathfrak{s o}(2, n)$, it is immediately clear that the $\mathrm{SO}(2, n)$-orbit type of $\alpha_{\Phi}$ at $p$ is constant all over $M$.

Now let us assume that the zero set $\operatorname{zero}(\varphi)$ of the non-trivial conformal Killing spinors $\varphi$ is non-empty on $(M, g)$. Let $p \in \operatorname{zero}(\varphi)$ be such a zero of $\varphi$. It follows immediately from (4) and (5) that $d \alpha_{\varphi}(p)=0$ and $d^{*} \alpha_{\varphi}(p)=0$. Also note that $\square \alpha_{\varphi}$ cannot vanish at $p$, since otherwise the 2-form tractor $\alpha_{\Phi}$ had to be constant zero on $M$. This shows that $\alpha_{\Phi}$ is given at $p \in M$ by

$$
\alpha_{\Phi}(p)=s_{+}^{b}(p) \wedge \square \alpha_{\varphi}(p),
$$

and this expression determines the orbit type of $\alpha_{\Phi}$ not only at $p$, but everywhere on $M$. In fact, (6) shows that the orbit type is represented by a simple wedge product $l \wedge c$ of a null 1 -form $l \in \mathbb{R}^{2, n^{*}}$ with another 1-form $c \neq 0$. Since this 1-form $c$ corresponds to the Dirac current of the spinor $\mathcal{D} \varphi$, it has to be causal. We conclude that the 2 -form tractor $\alpha_{\Phi}$, which belongs via the twistor $\Phi$ to the conformal Killing spinor $\varphi \in \Gamma(\mathcal{S})$ with zero at $p$, has two possible normal forms in $\Lambda^{2}\left(\mathbb{R}^{2, n^{*}}\right)$ under the adjoint action of $\mathrm{SO}(2, n)$, namely either $l \wedge c$ with $c$ null or $l \wedge c$ with $c$ timelike!

Moreover, let us say that $V_{\varphi}$ is hypersurface orthogonal if the $g$-orthogonal complement $V_{\varphi}^{\perp} \subset$ $T M$ of $V_{\varphi}$ is an integrable distribution of codimension 1 in $T M\left(\right.$ over $M \backslash \operatorname{zero}\left(V_{\varphi}\right)$ ).

Lemma 1. Let $\varphi \in \Gamma(\mathcal{S})$ be a non-trivial conformal Killing spinor on a Lorentzian spin manifold $\left(M^{n}, g\right)$ of dimension $n \geq 3$ with $\operatorname{zero}(\varphi) \neq \varnothing$. Then the Dirac current $V_{\varphi}$ of $\varphi$ is hypersurface orthogonal on $M \backslash \operatorname{zero}(\varphi)$.

Proof. Since $\alpha_{\Phi}$ is simple, we have $\alpha_{\Phi} \wedge \alpha_{\Phi}=0$ on $(M,[g])$. In particular, this proves $\alpha_{\varphi} \wedge d \alpha_{\varphi}=0$ on $M$. It follows directly by Frobenius' theorem that the annihilator $\left\{X \in T M \mid \iota_{X} \alpha_{\varphi}=0\right\}$ is an integrable distribution of codimension 1 on $M \backslash \operatorname{zero}(\varphi)$. The annihilator is the $g$-orthogonal complement of $V_{\varphi}$ in $T M$.

It is a well known fact that any hypersurface orthogonal, conformal Killing vector field $V$, which does not change its causal type, is (at least locally) parallel with respect to the Levi-Civita connection $\nabla^{\tilde{g}}$ of some metric $\tilde{g}$ in the given conformal class $c=[g]$ of a Lorentzian manifold $(M, g)$ (cf. e.g. [20]). We call a Lorentzian metric $\tilde{g}$, which admits a parallel null vector field, a Brinkmann metric.

Proposition 1. Let $\varphi \not \equiv 0$ be a conformal Killing spinor on a connected Lorentzian spin manifold $\left(M^{n}, g\right)$ of dimension $n \geq 3$ with $\operatorname{zero}(\varphi) \neq \varnothing$. Then the set $M \backslash \operatorname{sing}(\varphi)$ is dense in $M$, and for any $q \notin \operatorname{sing}(\varphi)$ there exists a neighbourhood $U_{q} \subset M \backslash \operatorname{sing}(\varphi)$ and a function $f$ on $U_{q}$ such that the rescaled spinor $\tilde{\varphi}:=e^{-f / 2} \varphi$ and the Dirac current $V_{\varphi}=V_{\tilde{\varphi}}$ are parallel with respect to the metric $\tilde{g}:=e^{2 f} g$ on $U_{q}$. Two cases are possible.

1. The Dirac current $V_{\varphi}$ is timelike in some point of $M$ and the Lorentzian metric $\tilde{g}$ on $U_{q}$ is isometric to a static monopole $-d t^{2}+h$, where $V_{\varphi}=\partial t$ and $h$ is a Ricci-flat Riemannian metric with parallel spinor.

2. The Dirac current $V_{\varphi}$ is null on $M \backslash \operatorname{zero}(\varphi)$ and $\tilde{g}$ on $U_{q}$ is a Brinkmann metric with parallel spinor. 
Proof. First, let us assume that $f$ is some function on an open subset $U$ in $M$ such that $V_{\varphi}$ is parallel with respect to the Levi-Civita connection $\nabla^{\tilde{g}}$ of $\tilde{g}=e^{2 f} g$. Then the 2-form tractor $\alpha_{\Phi}$ to $\tilde{\varphi}=e^{-f / 2} g$ must be given on $U$ with respect to $\tilde{g}$ by $s_{-}^{b} \wedge \alpha_{\tilde{\varphi}}+s_{+}^{b} \wedge \square^{\tilde{g}} \alpha_{\tilde{\varphi}}$. However, since the orbit type of $\alpha_{\Phi}$ is $l \wedge c$ with $c$ causal, we can conclude $\square^{\tilde{g}} \alpha_{\tilde{\varphi}}=0$ and $\alpha_{\Phi}=s_{-}^{b} \wedge \alpha_{\tilde{\varphi}}$. This shows $\mathcal{D}^{\tilde{g}} \tilde{\varphi}=0$ on $U$. Hence $\tilde{\varphi}$ is a parallel spinor on $(U, \tilde{g})$.

Next we see that if $V_{\varphi}$ is timelike at $p \in M$, then $V_{\varphi}$ cannot be null on an open subset $W$ of $M$. (Otherwise the 1 -form $c$ of the orbit type of $\alpha_{\Phi}$ would be null on $W$ and timelike at $p$.) Hence, since $M \backslash \operatorname{zero}(\varphi)$ is dense in $M$, we can also conclude that $M \backslash \operatorname{sing}(\varphi)$ is dense in $M$.

It remains to describe the local conformal geometry of $g$ off the singularity set on $M$. First, let us assume that $V_{\varphi}$ is timelike on $M \backslash \operatorname{sing}(\varphi)$. In this case we can rescale to $\tilde{g}:=\left(g\left(V_{\varphi}, V_{\varphi}\right)\right)^{-2} \cdot g$ on $M \backslash \operatorname{sing}(\varphi)$. Since $V_{\varphi}$ is parallel with respect to $\tilde{g}$, there exits locally a coordinate $t$ such that $\tilde{g}$ is given by $-d t^{2}+h$ with some Riemannian metric $h$. It is well known in this case that the $\tilde{g}$-parallel spinor $\tilde{\varphi}$ induces a parallel spinor for the Riemannian metric $h$. In particular, $h$ is Ricci-flat and $\tilde{g}=-d t^{2}+h$ is a static monopole metric.

In the other case, the Dirac current $V_{\varphi}$ is null and locally parallel with respect to some metric $\tilde{g}=e^{2 f} g$ on an appropriate neighbourhood $U_{q}$ of any point $q \in M \backslash \operatorname{zero}(\varphi)$. This means that $\tilde{g}$ is a Brinkmann metric. The parallel spinor is $\tilde{\varphi}:=e^{-f / 2} \varphi$ on $U_{q}$.

Remark 1. We want to emphasis here that the arguments in this section essentially rely on tractor calculus. The assumption $\operatorname{zero}(\varphi) \neq \varnothing$ in Lemma 1 and Proposition 1 determines via tractor calculus the orbit type of $\alpha_{\Phi}$, and hence the conformal geometry not only next to the zero set, but everywhere on $M \backslash \operatorname{sing}(\varphi)$. Note that, in general, a conformal Killing spinor without zero on a Lorentzian spin manifold is neither conformally related to a parallel spinor nor is its Dirac current hypersurface orthogonal. In fact, certain conformal Killing spinors on Lorentzian Einstein-Sasaki spaces do not have this property (cf. e.g. [3]).

Also note that an analogous result in Riemannian geometry is well known. Any conformal Killing spinor $\varphi$ with zero on a Riemannian manifold $(N, h)$ is conformally related to a parallel spinor off its zero set. A proof of this result does not need tractor calculus. One can rescale the Riemannian metric $h$ with $\|\varphi\|^{-4}$, where $\|\varphi\|$ is the spinor norm, and argue that the rescaled spinor $\tilde{\varphi}=\frac{1}{\|\varphi\|} \varphi$ is parallel (cf. [5]). In Lorentzian geometry this kind of proof does not work, alone for the simple reason that the Hermitian product $\langle\cdot, \cdot\rangle_{S}$ on the spinor bundle is indefinite. It seems that a proof with tractor methods is unavoidable in the Lorentzian case.

\section{The shape of the zero set}

Finally, we discuss here the shape of the zero set $\operatorname{zero}(\varphi)$ and the $\operatorname{singular} \operatorname{set} \operatorname{sing}(\varphi)$ of a conformal Killing spinor $\varphi$ on a Lorentzian spin manifold. The results of this section are based on arguments from [15] and [16].

Let $\left(M^{n}, g\right), n \geq 3$, be a Lorentzian spin manifold with spinor bundle $\mathcal{S}$. The spinor squaring has the following useful properties.

Lemma $2([16,6])$. Let $\varphi \in \mathcal{S}_{p}$ be a non-trivial spinor at $p \in M$ with spinor square $V_{\varphi} \in T_{p} M$. Then

1. $V_{\varphi}$ is causal.

2. $X \cdot \varphi=0$ if and only if $V_{\varphi}$ is null and $X \in \mathbb{R} V_{\varphi}$.

In the following, we denote by $\gamma_{p}: I=(a, b) \rightarrow M, a<0<b \in \mathbb{R}$, a geodesic of the Lorentzian manifold $(M, g)$ with $\gamma_{p}(0)=p$. We call a geodesic $\gamma_{p}$ maximal on $M$ if the interval $I$ is the maximal domain of definition. For an arbitrary point $p$ of the Lorentzian manifold $(M, g)$, we call the set $L_{p}$ of those points in $M$, which can be joined with $p$ by a smooth null geodesic, the geodesic null cone with origin $p$. We have the following general result from [15] (see also [16, 11]). 
Lemma 3. Let $V$ be a conformal vector field on a Lorentzian manifold $\left(M^{n}, g\right)$ with $\nabla^{g} V(p)=0$ for all $p \in \operatorname{zero}(V)$. Then

1. There exists for any $p \in \operatorname{zero}(V)$ an open neighbourhood $U_{p} \subset M$ such that $\operatorname{zero}(V) \cap U_{p}$ is contained in the image $\operatorname{Im}\left(\gamma_{p}\right)$ of a smooth null geodesic $\gamma_{p}$.

2. For any point $q \in L_{p}$ of the null cone of $p \in \operatorname{zero}(V)$, the vector $V(q) \in T_{q} M$ is a multiple of the tangent vector to a null geodesic, which joins $p$ and $q$. In particular, any $V(q) \neq 0$ is null for $q \in L_{p}$ and $p \in \operatorname{zero}(V)$.

Now let $\varphi \in \Gamma(\mathcal{S})$ be a conformal Killing spinor on $\left(M^{n}, g\right)$ with $\operatorname{zero}(\varphi) \neq \varnothing$. Note that the relations (4) and (5) show $\left(\nabla^{g} V_{\varphi}\right)(p)=0$ for any $p \in \operatorname{zero}(\varphi)$, i.e., Lemma 3 is applicable for the Dirac current $V_{\varphi}$ of $\varphi$.

Lemma 4. Let $\varphi \in \Gamma(\mathcal{S})$ be a conformal Killing spinor with zero on a Lorentzian spin manifold $\left(M^{n}, g\right)$, and let $\gamma_{p}$ be a geodesic on $M$ with $\gamma_{p}(0)=p$.

1. If $\gamma_{p}^{\prime}(0) \cdot \mathcal{D} \varphi(p)=0$ then $\operatorname{Im}\left(\gamma_{p}\right) \subset \operatorname{zero}(\varphi)$.

2. If $\gamma_{p}^{\prime}(0) \cdot \mathcal{D} \varphi(p) \neq 0$ then there exists a neighbourhood $U_{p}$ of $p$ in $M$ such that $p$ is the only zero of $\varphi$ in the image of $\gamma_{p}$ in $U_{p}$.

Proof. Let $\mathcal{B}(t)=\left(b_{1}(t), \ldots, b_{m}(t)\right)$ be a $\nabla^{\mathcal{S}}$-parallel complex spinor frame along $\gamma_{p}(t)$. (The complex rank of $\mathcal{S}$ is $m:=2^{\left[\frac{n}{2}\right]}$.) We set $u_{i}(t):=\left\langle\varphi\left(\gamma_{p}(t)\right), b_{i}(t)\right\rangle_{\mathcal{S}}$ for $i=1, \ldots, m$. Then we have

$$
u_{i}^{\prime}=-\frac{1}{n}\left\langle\gamma_{p}^{\prime} \cdot \mathcal{D} \varphi, b_{i}\right\rangle_{\mathcal{S}} \quad \text { and } \quad u_{i}^{\prime \prime}=-\frac{1}{2}\left\langle\gamma_{p}^{\prime} \mathrm{P}\left(\gamma_{p}^{\prime}\right) \cdot \varphi, b_{i}\right\rangle_{\mathcal{S}},
$$

i.e., the complex vector $U(t):=\left(\begin{array}{c}u_{1}(t) \\ \vdots \\ u_{m}(t)\end{array}\right)$ satisfies the ordinary differential equation $U^{\prime \prime}=$ $-\frac{1}{2} C \cdot U$, where $C \in M(m, \mathbb{C})$ is the complex matrix of the endomorphism $\varphi \in \mathcal{S} \mapsto \gamma_{p}^{\prime} \mathrm{P}\left(\gamma_{p}^{\prime}\right) \cdot \varphi \in \mathcal{S}$ with respect to $\mathcal{B}(t)$. By assumption, we have $U(0)=0$. Moreover, $U^{\prime}(0)=0$ if and only if $\gamma_{p}^{\prime}(0) \cdot \mathcal{D} \varphi(p)=0$. Obviously, the general theory of ordinary differential equations implies the two statements of Lemma 4.

We obtain the following result about the shapes of $\operatorname{zero}(\varphi)$ and $\operatorname{sing}(\varphi)$.

Proposition 2. Let $\varphi \in \Gamma(\mathcal{S})$ be a non-trivial conformal Killing spinor with $\operatorname{zero}(\varphi) \neq \varnothing$ on a connected Lorentzian spin manifold $\left(M^{n}, g\right)$.

1. If $V_{\varphi}$ is null on $M \backslash \operatorname{zero}(\varphi)$, then $\operatorname{sing}(\varphi)=\operatorname{zero}(\varphi)$ is a countable union of isolated images of smooth maximal null geodesics.

2. If $V_{\varphi}$ is timelike at some point $p \in M$, then $\operatorname{zero}(\varphi)$ is a countable union of isolated points. The singular set $\operatorname{sing}(\varphi)$ contains all geodesic null cones $L_{p}$ with origin $p \in \operatorname{zero}(\varphi)$. In some neighbourhood $U_{p} \subset M$ of any $p \in \operatorname{zero}(\varphi)$, the singular set $\operatorname{sing}(\varphi)$ equals the null cone $U_{p} \cap L_{p}$.

Proof. (1) First, let us assume that $V_{\varphi}$ is null on $M \backslash \operatorname{zero}(\varphi)$. Then we know from (6) that $V_{\mathcal{D} \varphi}(p)$ is null in $p \in \operatorname{zero}(\varphi)$. By Lemma 2 and Lemma $4(1)$, we know that the image of the maximal null geodesic $\gamma_{p}$ with $\gamma_{p}^{\prime}(0)=V_{\mathcal{D} \varphi}(p)$ is contained in $\operatorname{zero}(\varphi)$. Now, Lemma 3 implies that in a neighbourhood $U_{p}$ of $p$ the zero set zero $(\varphi)$ is equal to $U_{p} \cap \operatorname{Im}\left(\gamma_{p}\right)$. However, since this is true for any zero $p$ of $\varphi$, the assertion (1) of Proposition 2 follows on a manifold with countable basis. 
(2) Let $V_{\varphi}$ be timelike at some point of $M$. Then from (6) we know that $V_{\mathcal{D} \varphi}(p)$ is timelike at $p \in \operatorname{zero}(\varphi)$. Hence, by Lemma $2, \gamma_{p}^{\prime}(0) \cdot \mathcal{D} \varphi(p)=0$ is impossible for any geodesic through $p \in \operatorname{zero}(\varphi)$, and Lemma 3 implies that the zero $p$ is an isolated point. This is true for any $p \in \operatorname{zero}(\varphi)$.

It remains to prove the statements about the $\operatorname{singular}$ set $\operatorname{sing}(\varphi)$. Obviously, Lemma $3(2)$ implies that $L_{p}$ is contained in $\operatorname{sing}(\varphi)$ for any $p \in \operatorname{zero}(\varphi)$. Now, let us assume that $V_{\varphi}(q)$ is null at a point $q$ in a small neighbourhood $U_{p}$ of $p$ with $q \notin L_{p}$. Since $V_{\varphi}$ is a conformal vector field, the integral curve to $V_{\varphi}$ through $q$ is a null curve in $M$. In fact, the integral curve to $V_{\varphi}$ through $q$ is a null (pre-)geodesic (cf. [16, p. 63]). However, a straightforward argument as in [15] shows that any maximal null geodesic, which runs through a point $q$ that is very close to $p$, has to intersect the null cone $L_{p}$ of $p$ in a point $\ell$. This intersection is transversal to $L_{p}$. Hence the Dirac current $V_{\varphi}$ has to be zero at $\ell$ (since $V_{\varphi}$ is tangent to two transverse null geodesics running through $\ell$ ). However, if we consider a point $q$, which is arbitrary close to $p$, then the intersection point $\ell$ is arbitrary close to $p$ as well. But $p$ is an isolated zero of $\varphi$. This implies that there cannot exist a point $q$ arbitrary close to $p \in \operatorname{zero}(\varphi)$ off the null cone $L_{p}$, where $V_{\varphi}$ is null.

In summary with Proposition 1 we obtain our main result.

Theorem 1. Let $\varphi \in \Gamma(\mathcal{S})$ be a non-trivial conformal Killing spinor with $\operatorname{zero}(\varphi) \neq \varnothing$ on a connected Lorentzian spin manifold $\left(M^{n}, g\right)$ of dimension $n \geq 3$. Then $\operatorname{zero}(\varphi)$ consists either of

1. isolated images of null geodesics and off the zero set the metric $g$ is locally conformally equivalent to a Brinkmann metric with parallel spinor, or

2. isolated points and off the singular set $\operatorname{sing}(\varphi)$ the metric $g$ is conformally equivalent to a static monopole $-d t^{2}+h$, where $h$ is a Riemannian metric with parallel spinor.

Remark 2. Both cases of Theorem 1 do occur. In fact, any conformal Killing spinor $\varphi$ on the Minkowski space $\mathbb{R}^{1, n-1}$ with a zero at the origin is explicitly given by

$$
\varphi: p \in \mathbb{R}^{1, n-1} \mapsto p \cdot S \quad \text { with } \quad S \in \triangle_{1, n-1} .
$$

For those $S \in \mathbb{\Delta}_{1, n-1}$, whose spinor square is null, the zero set of $\varphi$ is a null line in $\mathbb{R}^{1, n-1}$ (in direction of the spinor square). For those $S \in \mathbb{\Delta}_{1, n-1}$, whose spinor square is timelike, the zero set consists only of the origin, and the null cone of $\mathbb{R}^{1, n-1}$ is the singular set.

Moreover, note that in [19] we have explicitly constructed a conformal Killing spinor with an isolated zero on a Lorentzian spin manifold, which is not conformally flat in any neighbourhood of the isolated zero. However, this Lorentzian metric is not smooth, but only $C^{1}$-differentiable, along the singular set. On the other hand, in [11] it is stated that any analytic Lorentzian metric, admitting a causal conformal vector field with a zero, is globally conformally flat.

\section{References}

[1] Bailey T.N., Eastwood M.G., Gover A.R., Thomas's structure bundle for conformal, projective and related structures, Rocky Mountain J. Math. 24 (1994), 1191-1217.

[2] Baum H., Spin-Strukturen und Dirac-Operatoren über pseudoriemannschen Mannigfaltigkeiten, TeubnerTexte zur Mathematik, Vol. 41, Teubner-Verlag, Leipzig, 1981.

[3] Baum H., Twistor and Killing spinors in Lorentzian geometry, in Global Analysis and Harmonic Analysis (Marseille-Luminy, 1999), Sémin. Congr., Vol. 4, Soc. Math. France, Paris, 2000, 35-52. 
[4] Baum H., Conformal Killing spinors and the holonomy problem in Lorentzian geometry - a survey of new results, in Symmetries and Overdetermined Systems of Partial Differential Equations, IMA Vol. Math. Appl., Vol. 144, Springer, New York, 2008, 251-264.

[5] Baum H., Friedrich Th., Grunewald R., Kath I., Twistor and Killing spinors on Riemannian manifolds, Teubner-Texte zur Mathematik, Vol. 124, Teubner-Verlag, Stuttgart - Leipzig, 1991.

[6] Baum H., Leitner F., The twistor equation in Lorentzian spin geometry, Math. Z. 247 (2004), 795-812, math.DG/0305063.

[7] Čap A., Slovák J., Weyl structures for parabolic geometries, Math. Scand. 93 (2003), 53-90, math.DG/0001166.

[8] Čap A., Slovák J., Souček V., Invariant operators on manifolds with almost Hermitian symmetric structures. I. Invariant differentiation, Acta Math. Univ. Comenian. (N.S.) 66 (1997), 33-69, dg-ga/9503003.

[9] Čap A., Slovák J., Souček V., Bernstein-Gelfand-Gelfand sequences, Ann. of Math. (2) 154 (2001), 97-113, math.DG/0001164.

[10] D'Ambra G., Gromov M., Lectures on transformation groups: geomerty and dynamics, in Surveys in Differential Geometry (Cambridge, MA, 1990), Lehigh Univ., Bethlehem, PA, 1991, 19-111.

[11] Frances Ch., Causal conformal vector fields, and singularities of twistor spinors, Ann. Global Anal. Geom. 32 (2007), 277-295.

[12] Friedrich H., Twistor connection and normal conformal Cartan connection, General Relativity and Gravitation 8 (1977), 303-312.

[13] Kobayashi S., Transformation groups in differential geometry, Springer-Verlag, New York - Heidelberg, 1972.

[14] Lawson H.B., Michelsohn M.-L., Spin geometry, Princeton Mathematical Series, Vol. 38, Princeton University Press, Princeton, NJ, 1989.

[15] Leitner F., Zeros of conformal vector fields and twistor spinors in Lorentzian geometry, SFB288-Preprint No. 439, Berlin, 1999.

[16] Leitner F., The twisor equation in Lorentzian geometry, Dissertation HU Berlin, 2001, available at http://edoc.hu-berlin.de/docviews/abstract.php?lang=ger \\&id=10473.

[17] Leitner F., Normal conformal Killing forms, math.DG/0406316.

[18] Leitner F., Conformal Killing forms with normalisation condition, Rend. Circ. Mat. Palermo (2) Suppl. (2005), no. 75, 279-292.

[19] Leitner F., Twistor spinors with zero on Lorentzian 5-space, Comm. Math. Phys. 275 (2007), 587-605, math.DG/0602622.

[20] Leitner F., Applications of Cartan and tractor calculus to conformal and CR-geometry, Habilitationsschrift Uni Stuttgart, 2007, available at http://elib.uni-stuttgart.de/opus/volltexte/2009/3922/.

[21] Lewandowski J., Twistor equation in a curved spacetime, Classical Quantum Gravity 8 (1991), L11-L17.

[22] Lichnerowicz A., Killing spinors, twistor spinors and Hijazi inequality, J. Geom. Phys. 5 (1988), 1-18.

[23] Penrose R., MacCallum M.A.H., Twistor theory: an approach to the quantisation of fields and space-time, Phys. Rep. C 6 (1973), 241-315.

[24] Penrose R., Rindler W., Spinors and space-time, Vol. 2, Spinor and twistor methods in space-time geometry, Cambridge Monographs on Mathematical Physics, Cambridge University Press, Cambridge, 1986.

[25] Thomas T., The differential invariants of generalized spaces, Cambridge University Press, Cambridge, 1934. 